\title{
Communication
}

\section{Selective arylation/annulation cascade reactions of 2-alkynylanilines with diaryliodonium salts}

\author{
Ying Duan a,b,*, Yanliang Yang b, Xiaoyu Dai b, Dongmi Li ${ }^{b}$ \\ a College of Food and Drug, Luoyang Normal University, Luoyang 471934, Henan, China \\ b College of Chemistry and Chemical Engineering, Henan Key Laboratory of Function-Oriented Porous Materials, Luoyang Normal University, Luoyang \\ 471934, Henan, China
}

\section{A R T I C L E I N F O}

\section{Article history:}

Received 16 July 2016

Accepted 13 September 2016

Published 5 November 2016

\section{Keywords:}

Selective arylation

Annulation

Diaryliodonium salt

2-Alkynylaniline

$\mathrm{N}$-Aryl indole

Cascade reaction

\section{A B S T R A C T}

An efficient $\mathrm{Cu}$ catalyzed selective arylation/annulation cascade reaction of 2-alkynylanilines with diaryliodonium salts was developed. This reaction was selective to $N$-arylation instead of $C$-arylation, which provides a simple synthetic method for $N$-aryl indoles.

(C) 2016, Dalian Institute of Chemical Physics, Chinese Academy of Sciences. Published by Elsevier B.V. All rights reserved.
Diaryliodonium salts are used in versatile organic transformations because of their highly electron-deficient nature and excellent leaving-group ability [1]. As mild, nontoxic, and selective reagents, they are competent arylating agents in organic synthesis [2,3], especially in constructing C-C [4-13] and C-X [14-17] bonds. Among these, nucleophilic substitution reactions between diaryliodonium salts with nitrogen nucleophiles have received much attention [18-21]. Recently, Guant's group [7] reported aromatic electrophile equivalents generated from the combination of diaryliodonium salts and copper catalysts via the intermediacy of $\mathrm{Cu}(\mathrm{III})$-aryl species. These active species were considered to be responsible for the high activity of the diaryliodonium salts in Cu catalyzed reactions.

Indoles are one of the most ubiquitous heterocycles found in nature. Owing to their significant biological activity, indoles are central structural motifs in making numerous pharmacologi- cally important compounds [22]. Consequently, much attention has been paid to their preparation, which is a major research area in organic synthesis and many methods have been developed [23]. Meanwhile, the cyclization of 2-alkynylaniline derivatives has long been exploited for the construction of indoles $[24,25]$.

Although diaryliodonium salts have been applied to a number of arylation reactions with substrates possessing single nucleophilic group, the selective arylation of the substrates with two or more nucleophilic groups is still a challenging subject $[4,6]$. For example, it was disclosed that although $\mathrm{C}$-arylation and $\mathrm{N}$-arylation can be achieved by diaryliodonium salts, the selectivity is still a challenge. The key to solving this is how to exploit the difference in the nucleophilic groups. Here, we reported an example of a selective $\mathrm{N}$-arylation reaction of 2-alkynylanilines with diaryliodonium salts where subse-

\footnotetext{
* Corresponding author. Tel/Fax: +86-379-68618516; E-mail: duanying7@163.com

This work was supported by the Intercollegiate Key Scientific Research Projects of Henan Province (15A150018).

DOI: 10.1016/S1872-2067(16)62522-6 | http://www.sciencedirect.com/science/journal/18722067 | Chin. J. Catal., Vol. 37, No. 11, November 2016
} 


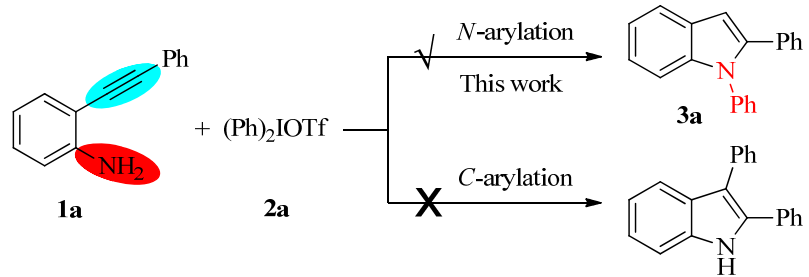

Scheme 1. Reaction of 2-alkynylaniline (1a) and diphenyliodonium salt (2a).

quently the untouched $C$-nucleophile behaved as another reactive site to complete arylation-annulation cascade reactions to afford $N$-aryl indoles [26-28] (Scheme 1).

We first examined the reaction of 2-(phenylethynyl)aniline (1a) and diphenyliodonium triflate (2a). First, to overcome the possible competition of $\mathrm{N}$-arylindoles and $\mathrm{C}$-arylation, 1,2-dichloroethane (DCE) was placed on the top of the solvent list because it has been demonstrated to favor $N$-aryltaion [4]. No reaction occurred when the metal catalyst was omitted. A $\mathrm{Cu}$ catalyst system has been shown to be effective in both the arylation and annulation of 2-alkynylanilines [29]. Both copper(I) and copper(II) salts were effective for this reaction, and the use of $10 \mathrm{~mol} \% \mathrm{Cu}\left(\mathrm{OCOC}_{8} \mathrm{H}_{17}\right)_{2}$ (copper 2-ethylhexoate) gave the desired product 3a in 93\% yield. Meanwhile, the use of $\mathrm{CuCl}, \mathrm{CuBr}, \mathrm{CuI}, \mathrm{CuSCN}$ and $\mathrm{Cu}(\mathrm{OTf})_{2}$ resulted in decreased yields (Table 1, entries 1-8). Then the screening of the base exhibited that $\mathrm{N}, \mathrm{N}$-diisopropylethylamine (DIPEA) was more effective than the other bases $\left(\mathrm{Et}_{3} \mathrm{~N}, \mathrm{~K}_{2} \mathrm{CO}_{3}, \mathrm{KOtBu}\right.$ and $\mathrm{K}_{3} \mathrm{PO}_{4}$, Table 1, entries 9-12). The influence of the solvent on the reac-

Table 1

Optimization of the reaction conditions.

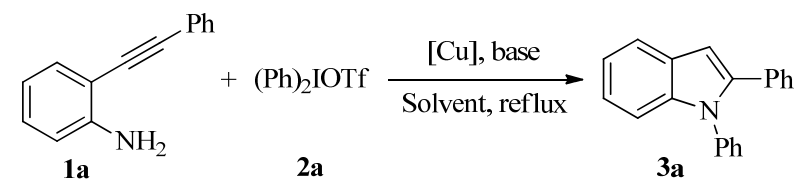

\begin{tabular}{|c|c|c|c|c|}
\hline Entry & {$[\mathrm{Cu}]$} & Base & Solvent & Yield a $(\%)$ \\
\hline 1 & - & DIPEA & DCE & $<5$ \\
\hline 2 & $\mathrm{CuCl}$ & DIPEA & DCE & 56 \\
\hline 3 & $\mathrm{CuBr}$ & DIPEA & DCE & 61 \\
\hline 4 & $\mathrm{CuI}$ & DIPEA & DCE & 67 \\
\hline 5 & CuSCN & DIPEA & DCE & 67 \\
\hline 6 & $\mathrm{CuBF}_{4}(\mathrm{MeCN})_{4}$ & DIPEA & DCE & 80 \\
\hline 7 & $\mathrm{Cu}(\mathrm{OTf})_{2}$ & DIPEA & DCE & 61 \\
\hline 8 & $\mathrm{Cu}\left(\mathrm{OCOC}_{8} \mathrm{H}_{17}\right)_{2}$ & DIPEA & DCE & 93 \\
\hline 9 & $\mathrm{Cu}\left(\mathrm{OCOC}_{8} \mathrm{H}_{17}\right)_{2}$ & $\mathrm{Et}_{3} \mathrm{~N}$ & DCE & 69 \\
\hline 10 & $\mathrm{Cu}\left(\mathrm{OCOC}_{8} \mathrm{H}_{17}\right)_{2}$ & $\mathrm{~K}_{2} \mathrm{CO}_{3}$ & DCE & $<5$ \\
\hline 11 & $\mathrm{Cu}\left(\mathrm{OCOC}_{8} \mathrm{H}_{17}\right)_{2}$ & $\mathrm{KO}^{t} \mathrm{Bu}$ & DCE & 26 \\
\hline 12 & $\mathrm{Cu}\left(\mathrm{OCOC}_{8} \mathrm{H}_{17}\right)_{2}$ & $\mathrm{~K}_{3} \mathrm{PO}_{4}$ & DCE & $<5$ \\
\hline 13 & $\mathrm{Cu}\left(\mathrm{OCOC}_{8} \mathrm{H}_{17}\right)_{2}$ & DIPEA & Toluene & 69 \\
\hline 14 & $\mathrm{Cu}\left(\mathrm{OCOC}_{8} \mathrm{H}_{17}\right)_{2}$ & DIPEA & $\mathrm{CH}_{2} \mathrm{Cl}_{2}$ & 56 \\
\hline 15 & $\mathrm{Cu}\left(\mathrm{OCOC}_{8} \mathrm{H}_{17}\right)_{2}$ & DIPEA & $\mathrm{CHCl}_{3}$ & 35 \\
\hline 16 & $\mathrm{Cu}\left(\mathrm{OCOC}_{8} \mathrm{H}_{17}\right)_{2}$ & DIPEA & THF & 20 \\
\hline $17 \mathrm{~b}$ & $\mathrm{Cu}\left(\mathrm{OCOC}_{8} \mathrm{H}_{17}\right)_{2}$ & DIPEA & DCE & 22 \\
\hline $18^{c}$ & $\mathrm{Cu}\left(\mathrm{OCOC}_{8} \mathrm{H}_{17}\right)_{2}$ & DIPEA & DCE & 48 \\
\hline $19 \mathrm{~d}$ & $\mathrm{Cu}\left(\mathrm{OCOC}_{8} \mathrm{H}_{17}\right)_{2}$ & DIPEA & DCE & 43 \\
\hline
\end{tabular}

Reaction conditions: 1a $(0.2 \mathrm{mmol}), 2 a(0.4 \mathrm{mmol}),[\mathrm{Cu}](10 \mathrm{~mol} \%)$,

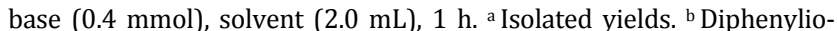
donium chloride used. c Diphenyliodonium hexafluorophosphate used. d Diphenyliodonium tetrafluoroborate used. tion was also examined. The result showed that DCE was the best choice while a decreased yield of the desired product was observed in solvents such as toluene, $\mathrm{CH}_{2} \mathrm{Cl}_{2}, \mathrm{CHCl}_{3}$ and THF (Table 1, entries 13-16). The counter-ions of the diphenyliodonium salt were also evaluated. The result showed that diphenyliodonium traflate was superior to diphenyliodonium chloride, diphenyliodonium hexafluorophosphate and diphenyliodonium tetrafluoroborate (Table 1, entries 17-19).

With these optimized reaction conditions in hand, we next elucidated the substrate scope of the cascade reaction. First, various $o$-alkynylanilines $\mathbf{1}$ with diphenyliodonium triflate (2a) were employed in the reaction. The results are compiled in Scheme 2. Generally, the reactions proceeded smoothly to afford the corresponding indoles $\mathbf{3}$ in isolated yields of $79 \%-97 \%$. The reaction went well when $\mathrm{R}^{1}=$ aryl group while the electronic nature of the substituent on the benzene ring of $\mathrm{R}^{1}$ was varied. Both electron-withdrawing and electron-donating groups were tolerated. Notably, it was found that alkynes bearing alkenyl $\left(\mathrm{R}^{1}=\right.$ alkenyl group) or alkyl groups $\left(\mathrm{R}^{1}\right.$ $=$ alkyl group) on the alkyne terminus were also compatible, providing the products $\mathbf{3 e - 3 g}$ in good to excellent yields of $79 \%-92 \%$. Substitution with 4-Me or 4-Cl functionalities on the parent aryl ring also afforded $\mathbf{3 h}$ and $\mathbf{3 i}$ in high yields of $89 \%$ and $97 \%$, respectively.

The scope of the reaction was also investigated by varying the diaryliodonium triflate. The results are summarized in Scheme 3. Interestingly, the sterically demanding di(o-methylpheny)liodonium triflate $\mathbf{2 b}$ gave product $\mathbf{4 b}$ in good yield (82\%). Higher yields (4c-4f) were obtained with less sterically demanding diaryliodonium triflates. Also, the substituent nature of $\mathrm{R}^{1}$ has some influence on the reaction (4f vs $\mathbf{4 g}$ ). Substrates with substituent on the benzene ring (1h) reacted with diaryliodonium triflate to afford $\mathbf{4 h}$ with good to excellent yields ( $82 \%$ and $98 \%$, respectively).

The reaction of $\mathbf{1 a}$ and unsymmetric diaryliodonium salt $\mathbf{2} \mathbf{i}$ was also evaluated. Aryl with a lesser steric effect could be transferred to $\mathbf{1 a}$, and $\mathbf{4 i}$ was obtained in $80 \%$ yield (Scheme 4).

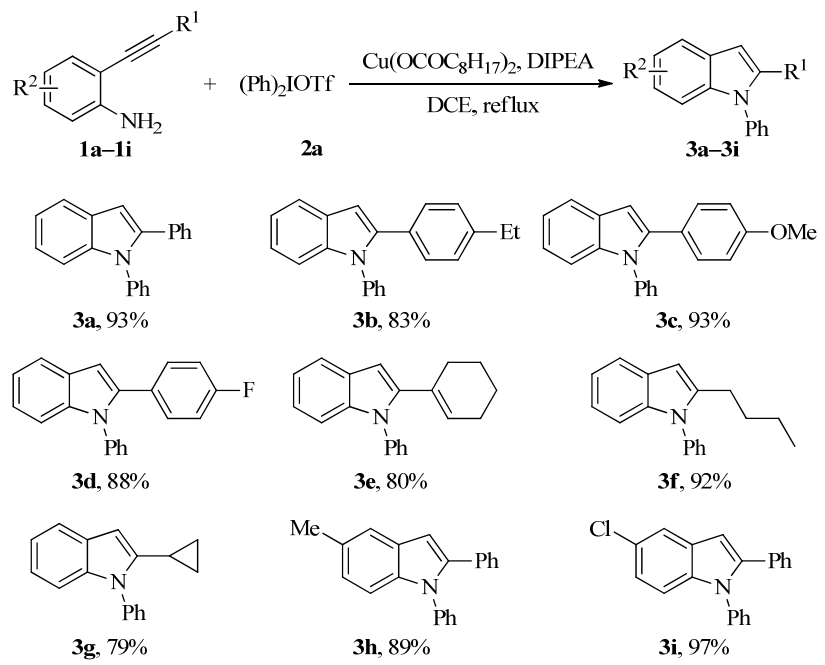

Scheme 2. Synthesis of $N$-phenyl indoles (3a-3i). Reaction conditions: 1 (0.2 mmol), 2a (0.4 mmol), $\mathrm{Cu}\left(\mathrm{OCOC}_{8} \mathrm{H}_{17}\right)_{2}(10 \mathrm{~mol} \%)$, DIPEA (0.4 $\mathrm{mmol})$, solvent $(2.0 \mathrm{~mL}), 1 \mathrm{~h}$. 
<smiles></smiles>

$4 b, 82 \%$<smiles>Fc1ccc(-n2c(-c3ccccc3)cc3ccccc32)cc1</smiles>

$+\mathrm{Ar}_{2} \mathrm{IOTf}$ $2 \mathrm{~b}-2 \mathrm{~h}$ $\underset{\text { DIPEA, DCE, reflux }}{\stackrel{\mathrm{Cu}\left(\mathrm{OCOC}_{8} \mathrm{H}_{17}\right)_{2}}{\longrightarrow}}$<smiles>[18OH]c1cc2ccccc2n1-c1ccccc1</smiles>
4c, $96 \%$ Me<smiles></smiles><smiles>c1ccc2[nH]ccc2c1</smiles>
4e, $71 \% \mathrm{Br}$<smiles>Fc1ccc(-n2c(-c3ccc(Br)cc3)cc3ccccc32)cc1</smiles>

Scheme 3. Synthesis of $N$-arylindole $(\mathbf{4 b}-\mathbf{4 h})$. Reaction conditions: $\mathbf{1}$ (0.2 mmol), 2 (0.4 mmol), $\mathrm{Cu}\left(\mathrm{OCOC}_{8} \mathrm{H}_{17}\right)_{2}(10 \mathrm{~mol} \%)$, DIPEA (0.4 mmol) solvent $(2.0 \mathrm{~mL}), 1 \mathrm{~h}$.<smiles>C#Cc1ccccc1N</smiles>

1a

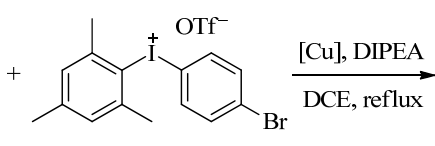

$2 \mathbf{i}$

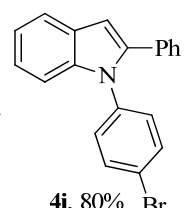

$4 \mathbf{4}, 80 \% \mathrm{Br}$
Scheme 4. Synthesis of $N$-arylindole (4i).

To probe the reaction mechanism, controlled experiments were performed. First, the reaction was operated with omitting of the diaryliodonium triflate, and the material did not react. When $\mathbf{5}$ was subjected to the reaction under the standard reaction conditions, the desired product 3a was obtained in $94 \%$ yield (Scheme 5).

According to these results, the cascade reaction can be described as follows (Scheme 6). First, the $\mathrm{Cu}$ catalyst and diaryliodonium salt generated $\mathrm{Cu}(\mathrm{III})-$ aryl, which was demonstrated to be a reactive intermediate. Then this reactive intermediate, which served as an aromatic electrophile, reacted with 2-alkynylaniline to give the $N$-arylation product (5) with the assistance of the base. Finally, heteroannulation took place with the help of the $\mathrm{Cu}$ catalyst to afford $\mathrm{N}$-arylindole (3).

In conclusion, we developed an efficient $\mathrm{Cu}$ catalyzed selective arylation/annulation cascade reaction of 2-alkynylanilines with diaryliodonium salts. The novelty of this strategy lies in giving excellent selectivity of $\mathrm{N}$-arylation as opposed to $C$-arylation. Many functional group and diaryliodonium salts

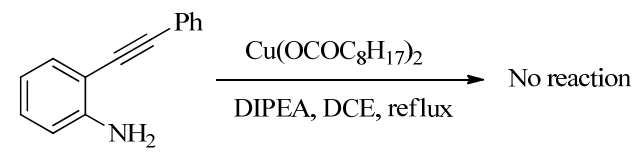<smiles>C(#Cc1ccccc1Nc1ccccc1)c1ccccc1</smiles>

5

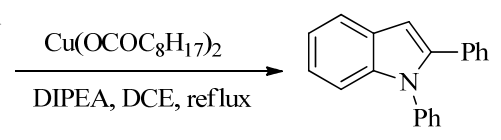

3a, $94 \%$
Scheme 5. Control experiments study.

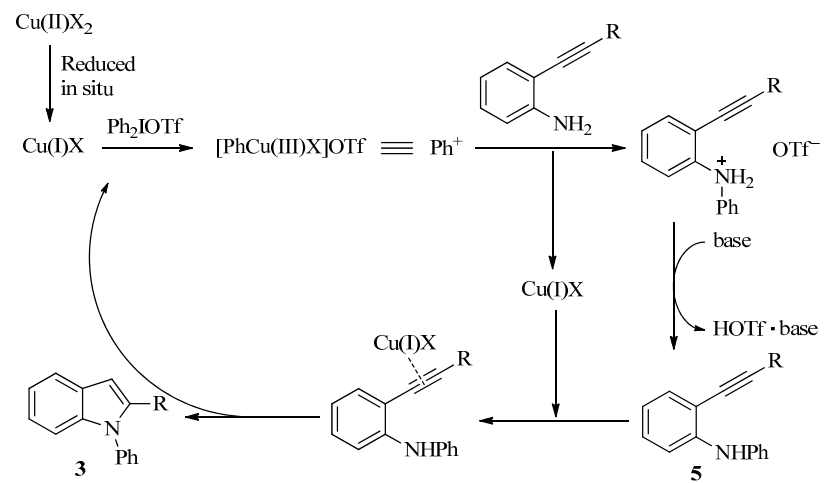

Scheme 6. Mechanism of the cascade reaction.

were tolerated to afford a large scope of $N$-aryl indoles with good to excellent yield. The reaction was shown to be the selective $\mathrm{N}$-arylation first and then heteroannulation.

\section{Acknowledgments}

The authors thank Prof. Yonggui Zhou and Dr. Wenxue Huang for chemical reagents and helpful discussions.

\section{References}

[1] V. V. Zhdankin, P. J. Stang, Chem. Rev., 2008, 108, 5299-5358.

[2] E. A. Merritt, B. Olofsson, Angew. Chem. Int. Ed., 2009, 48, 9052-9070.

[3] Z. C. Xiao, C. F. Xia, Chin. J. Org. Chem., 2013, 33, 2119-2130.

[4] S. G. Modha, M. F. Greaney, J. Am. Chem. Soc., 2015, 137, 1416-1419.

[5] D. Holt, M. J. Gaunt, Angew. Chem. Int. Ed., 2015, 54, 7857-7861.

[6] E. Cahard, H. P. J. Male, M. Tissot, M. J. Gaunt, J. Am. Chem. Soc., 2015, 137, 7986-7989.

[7] F. Z. Zhang, S. Das, A. J. Walkinshaw, A. Casitas, M. Taylor, M. G. Suero, M. J. Gaunt, J. Am. Chem. Soc., 2014, 136, 8851-8854.

[8] Y. Yang, J. W. Han, X. S. Wu, S. Mao, J. J. Yu, L. M. Wang, Synlett, 2014, 25, 1419-1424.

[9] Y. Wang, C. Chen, J. Peng, M. Li, Angew. Chem. Int. Ed., 2013, 52, 5323-5327.

[10] A. J. Walkinshaw, W. S. Xu, M. G. Suero, M. J. Gaunt, J. Am. Chem. Soc., 2013, 135, 12532-12535.

[11] Q. Y. Toh, A. McNally, S. Vera, N. Erdmann, M. J. Gaunt, J. Am. Chem. Soc., 2013, 135, 3772-3775.

[12] M. G. Suero, E. D. Bayle, B. S. L. Collins, M. J. Gaunt, J. Am. Chem. Soc., 2013, 135, 5332-5335.

[13] B. S. L. Collins, M. G. Suero, M. J. Gaunt, Angew. Chem. Int. Ed., 2013, 52, 5799-5802.

[14] W. S. Guo, S. L. Li, L. Tang, M. Li, L. R. Wen, C. Chen, Org. Lett., 2015, $17,1232-1235$.

[15] L. Chan, A. McNally, Q. Y. Toh, A. Mendoza, M. J. Gaunt, Chem. Sci., 2015, 6, 1277-1281.

[16] B. Bhattarai, J. H. Tay, P. Nagorny, Chem. Commun., 2015, 51, 5398-5401.

[17] S. Mao, F. L. Guo, J. Li, X. Geng, J. J. Yu, J. W. Han, L. M. Wang, Synlett, 2013, 24, 1959-1962.

[18] X. Geng, S. Mao, L. S. Chen, J. J. Yu, J. W. Han, J. L. Hua, L. M. Wang, Tetrahedron Lett., 2014, 55, 3856-3859.

[19] Y. Wang, C. Chen, S. Zhang, Z. B. Lou, X. Su, L. R. Wen, M. Li, Org. Lett., 2013, 15, 4794-4797. 


\title{
Graphical Abstract
}

Chin. J. Catal., 2016, 37: 1837-1840 doi: 10.1016/S1872-2067(16)62522-6

Selective arylation/annulation cascade reactions of 2-alkynylanilines with diaryliodonium salts

Ying Duan *, Yanliang Yang, Xiaoyu Dai, Dongmi Li Luoyang Normal University

An efficient $\mathrm{Cu}$ catalyzed selective arylation/annulation cascade reaction of 2-alkynylaniline with diaryliodonium salts was developed.

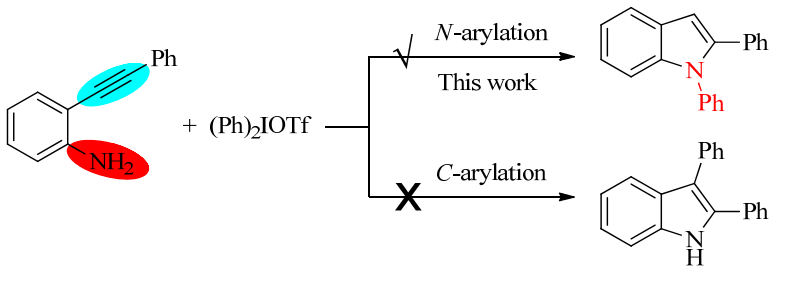

[20] X. Su, C. Chen, Y. Wang, J. J. Chen, Z. B. Lou, M. Li, Chem. Commun., 2013, 49, 6752-6754.

[21] J. Peng, C. Chen, Y. Wang, Z. B. Lou, M. Li, C. J. Xi, H. Chen, Angew. Chem. Int. Ed., 2013, 52, 7574-7578.

[22] K. Higuchi, T. Kawasaki, Nat. Prod. Rep., 2007, 24, 843-868.

[23] M. Inman, C. J. Moody, Chem. Sci., 2013, 4, 29-41.

[24] K. Krueger, A. Tillack, M. Beller, Adv. Synth. Catal., 2008, 350, 2153-2167.
[25] G. R. Humphrey, J. T. Kuethe, Chem. Rev., 2006, 106, 2875-2911.

[26] S. C. Song, M. N. Huang, W. J. Li, X. H. Zhu, Y. Q. Wan, Tetrahedron, 2015, 71, 451-456.

[27] L. Yang, Y. H. Ma, F. J. Song, J. S. You, Chem. Commun., 2014, 50, 3024-3026.

[28] J. Sheng, S. Li, J. Wu, Chem. Commun., 2014, 50, 578-580.

[29] J. Ezquerra, C. Pedregal, C. Lamas, J. Barluenga, M. Pérez, M. A. García-Martín, J. M. González, J. Org. Chem., 1996, 61, 5804-5812.

\section{2-炔基苯胺与二芳基碘鎓盐的选择性芳基化/环化串联反应}

\author{
段 英 ${ }^{\mathrm{a}, \mathrm{b},{ }^{*}}$, 杨艳良 ${ }^{\mathrm{b}}$, 戴晓玉 ${ }^{\mathrm{b}}$, 李东密 ${ }^{\mathrm{b}}$

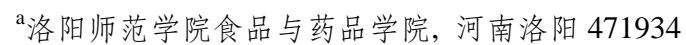

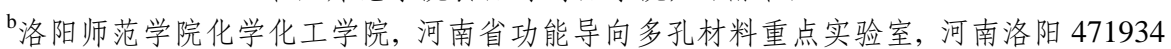

摘要: 有机高价碘试剂是一类环境友好、制备简单且性质温和的有机合成新试剂. 近年来, 有机高价碘试剂因表现出新颖、 独特的反应性能而受到化学工作者广泛关注, 成为有机合成重要研究领域之一. 二芳基碘鈆盐是有机高价碘试剂的一个 重要组成部分, 是一类具有较高普适性的芳基化试剂, 可用于羰基化合物、烯烃、炔烃和杂原子亲核化合物等的芳基化反 应. 目前, 二芳基碘鍮盐作为芳基化试剂对具有单一芳基化位点化合物的芳基化已经有了非常广泛而深入的研究. 对于具 有两个甚至多个芳基化位点的化合物 (如同时具有胺基和炔基), 其芳基化选择性问题仍缺乏系统研究. 特别是在多个芳基 化位点共存时如何能够使芳基化发生在某一特定位点仍然是一大难题. 这限制了二芳基碘鉾盐作为芳基化试剂更广泛的 应用. 因此, 我们选用 2-炔基苯胺(含有胺基和炔基两个芳基化位点)作为原料, 通过溶剂的选择以及溶液酸碱性的调控来 改变不同芳基化位点的反应活性, 通过催化剂的调变来改变二芳基碘鍮盐芳基化反应的能力, 从而找出最优条件实现底物 分子的选择性芳基化反应, 并利用剩余活性位点实现分子内的环化反应, 从而实现芳基化-环化串联反应合成一系列 $N$-芳 基吲哚类化合物.

在对模型底物进行条件篮选实验时发现, 以 2-乙基辛酸铜 $\left(\mathrm{Cu}\left(\mathrm{OCOC}_{8} \mathrm{H}_{17}\right)_{2}\right)$ 作催化剂, 二异丙基乙基胺 (DIPEA) 作碱, 1,2 二氯乙烷 (DCE) 为溶剂, 反应以最高 $93 \%$ 的收率得到 1,2-二苯基吲哚. 使用该最优反应条件, 一系列 2-炔基苯胺都能 与二芳基碘鎓盐很好地发生反应并且以良好到优秀的产率 (71\%-98\%) 得到目标产物 $N$-芳基吲哚. 此外, 2-炔基苯胺与非 对称的二芳基碘鎓盐也能发生反应, 实验结果证明为位阻较小的芳基对胺基进行了 $\mathrm{N}$-芳基化反应. 通过空白实验和对比 实验, 我们提出了可能的反应机理: 二芳基碘鎓盐在铜催化剂作用下转化为亲电性的芳基活性中间体, 该中间体与底物的 胺基发生芳基化反应, 然后芳基化产物在铜催化剂作用下环化生成 $N$-芳基吲哚.

该反应很好地解决了同时具有胺基和炔基两个芳基化位点的底物与二芳基碘鎓盐反应时 $C$-芳基化和 $N$-芳基化的竞 争问题, 选择合适反应条件使 $N$-芳基化反应优先进行, 为二芳基碘鍮盐的选择性芳基化反应提供了很好的实例, 并为其它 具有多个芳基化位点化合物的选择性芳基化反应提供了途径.

关键词: 选择性芳基化; 二芳基碘鈆盐; 环化反应; 2 -炔基苯胺; $N$-芳基吲哚; 串联反应

收稿日期: 2016-07-16. 接受日期: 2016-09-13. 出版日期: 2016-11-05.

*通讯联系人. 电话/传真: (0379)68618516; 电子信箱: duanying7@163.com

基金来源: 河南省高等学校重点科研项目(15A150018).

本文的英文电子版由Elsevier出版社在ScienceDirect上出版(http://www.sciencedirect.com/science/journal/18722067). 